\title{
Flora da Bahia: Achariaceae
}

\section{Amanda Pricilla Batista Santos ${ }^{1 *}$, Luciano Paganucci de Queiroz ${ }^{1, a}$ \& André Márcio Araújo Amorim²,b}

${ }^{1}$ Departamento de Ciências Biológicas, Universidade Estadual de Feira de Santana, Feira de Santana, Bahia, Brasil.

${ }^{2}$ Departamento de Ciências Biológicas, Universidade Estadual de Santa Cruz, Ilhéus \& Herbário Centro de Pesquisas do Cacau, Itabuna, Bahia, Brasil.

Resumo - É apresentado o tratamento taxonômico de Achariaceae para o estado da Bahia, Brasil. Foram reconhecidos dois gêneros, com uma espécie cada: Carpotroche brasiliensis e Kuhlmanniodendron macrocarpum, esta última endêmica do estado. São apresentados chave de identificação, descrições, comentários taxonômicos, ilustrações e mapas de distribuição das espécies no estado.

Palavras-chave adicionais: florística, Mata Atlântica, Nordeste do Brasil, taxonomia.

Abstract (Flora of Bahia: Achariaceae) - The taxonomic treatment of the Achariaceae from Bahia state, Brazil, is presented. Two genera are recognized, with one species each: Carpotroche brasiliensis and Kuhlmanniodendron macrocarpum, this latter endemic to the state. Identification key, descriptions, taxonomic comments, illustrations and distribution maps of species in the state are presented.

Additional key words: Atlantic forest, floristics, northeastern Brazil, taxonomy.

\section{ACHARIACEAE}

Árvores a arbustos ou ervas escandentes. Folhas alternas dísticas ou espiraladas, simples, com ou sem estípulas. Flores monoclinas ou diclinas, actinomorfas, diclamídeas; cálice 2-5-mero, dialissépalo; corola 4-15-mera, dialipétala; estames 5 a numerosos, anteras rimosas, basifixas; disco nectarífero ausente; ovário súpero, (2)3-8(-10)-carpelar, unilocular, geralmente pluriovulado, placentação parietal, estiletes 1-8(-10). Inflorescências unifloras, fasciculadas, em racemos, panículas ou botrioides, axilares, subterminais ou caulifloras. Frutos bagas ou cápsulas, lisos ou ornamentados com verrugas, espinhos, cerdas ou alas verticais. Sementes 1 a numerosas, ariladas.

Achariaceae possui cerca de 30 gêneros e 150 espécies e apresenta distribuição pantropical. No Brasil, ocorrem quatro gêneros e cerca de 18 espécies (Marquete et al. 2015). No estado da Bahia, são registradas duas espécies.

\section{Chave para espécies}

1. Lâmina foliar com tricomas sobre a nervura principal da face abaxial; filetes hirsutos; estiletes ca. 6; bagas com epicarpo alado ... 1.1. Carpotroche brasiliensis

1'. Lâmina foliar glabra; filetes glabros; estiletes 3; bagas com epicarpo liso a tuberculado

\subsection{Kuhlmanniodendron macrocarpum}

\section{Carpotroche Endl.}

Arbustos ou árvores, poligâmicos, monoicos ou dioicos. Folhas alternas espiraladas; lâmina com

\footnotetext{
*Autora para correspondência: amanda.pricilla@hotmail.com; aluciano.paganucci@gmail.com; bamorim.uesc@gmail.com Editor responsável: Pedro Fiaschi

Submetido: 27 out. 2015; aceito: 27 abr. 2016

Publicação eletrônica: 21 jun. 2016; versão final: 29 jun. 2016
}

margem inteira a serrilhada; venação broquidódroma; estípulas lineares, lanceoladas, ovadas, triangulares ou subuladas, decíduas. Inflorescências em cimeiras axilares ou caulifloras. Flores estaminadas ou monoclinas; perianto assimetricamente arranjado. Sépalas (2)3. Pétalas (4-)6-9(-12), subseriadas. Estames numerosos; filetes densamente pilosos ou hirsutos a híspidos; anteras lineares, pilosas, pubescentes ou hirsutas. Ovário 4-10-carpelar, pluriovular, com (4-)6-16 alas verticais ou quase liso (com costelas inconspícuas); estiletes 4-8(-10), persistentes, conatos na base; estigmas subcapitados a capitados, lacerados. Frutos bagas ou cápsulas tardiamente deiscentes, com 4-8(-10) valvas; epicarpo alado, (4-)6-16 alas verticais (raramente com costelas pouco proeminentes), inteiras, crenadas ou laceradas. Sementes numerosas, obovoides a ovoide-compressas ou poliédricas; testa lisa.

Carpotroche possui ampla distribuição na América Central, da Guatemala ao Panamá, e na América do Sul, principalmente na Amazônia. Inclui 11 espécies; oito delas ocorrem no Brasil, nos domínios da Amazônia, Cerrado e Mata Atlântica (Sleumer 1980; Marquete et al. 2015).

1.1. Carpotroche brasiliensis (Raddi) A.Gray, U.S. Expl. Exped., Atlas Phan. 1: 72. 1854.

Figuras 1, 2A-D e 4.

Nomes populares: fruto-de-paca, inhaíba, jindiba, jindiba-branca.

Árvores ou arbustos até $15 \mathrm{~m}$ alt., polígamodioicos; tronco lenticelado, ramos glabrescentes ou tomentulosos a tomentosos quando jovens. Folhas com pecíolo 1-3 cm compr.; lâmina 9,5-28 × 3-12 cm, obovada a elíptica, base atenuada, cuneada ou obtusa, ápice agudo, acuminado, raramente obtuso, margem inteira a crenada ou serrilhada, cartácea a subcoriácea, 
verde-escura adaxialmente, verde-pálida a ferrugínea abaxialmente, tricomas esparsos restritos às nervuras, nervura principal serícea a tomentosa, nervuras principal e secundárias proeminentes em ambas as faces; estípulas lineares. Inflorescências estaminadas em cimeiras 1-3-floras, flores monoclinas em cimeiras unifloras; pedúnculo 2,5-6,5 cm compr., seríceo a glabrescente, brácteas e bractéolas $4-13 \mathrm{~mm}$ compr., lineares, pedicelo 1-2,2 cm compr., seríceo a tomentoso. Sépalas 3, 10-21 × 7-17 mm, obovadas a largamente elípticas, membranáceas, esverdeadas, seríceas externamente. Pétalas 7-11, 11-25 × 5-11 $\mathrm{mm}$, elípticas a obovadas, brancas, seríceas em ambas as faces. Estames ca. 150, hirsutos; filetes $4-5 \mathrm{~mm}$ compr., espessados; anteras 6-7 mm compr. Ovário ca. $8 \times 8 \mathrm{~mm}$; estiletes ca. 6 , estigmas capitados, lacerados. Bagas 10-15 $\times 5-8,5 \mathrm{~cm}$, elípticas a subglobosas, providas de alas longitudinais plicadas, membranáceas a papiráceas, adpressas ao epicarpo; endocarpo amarelado. Sementes ca. $2 \times 1,3 \mathrm{~cm}$.

Bahia, Minas Gerais, Espírito Santo e Rio de Janeiro, nos domínios do Cerrado e Mata Atlântica (Sleumer 1980; Marquete et al. 2015). D/E3, D/E9, D/E10, E8, E9, F8, F9, G8, G8/9, G/H8, H8/9, H/I8, I8/9, J8, K8: Mata Atlântica, em florestas ombrófilas montanas e florestas estacionais semideciduais. Floresce de agosto a maio e pode ser encontrada com frutos durante todo o ano.

Material selecionado - Alagoinhas, fev. 1998 (fr.), M.L. Guedes et al. in PL0320 (HUESC); Almadina, 14\% $41^{\prime} 28^{\prime \prime S}$, 39 36'35"W, nov. 2013 (fl.), L.C. Marinho et al. 591 (HUEFS); Amargosa, 1409'66"S, 3949'44"W, out. 2010 (fr.), J.L. Paixão \& M.S. Nascimento 1366 (HUEFS); Arataca, 15\%10'27"S, 39²0'22"W, fev. 2011 (fl.), P.M. Leitman et al. 97 (CEPEC); Barra do Rocha, 14¹0'52"S, 39³6'22"W, ago. 2001 (fr.), D.M. Loureiro et al. 604 (ALCB); Buerarema, 14 $57^{\circ} \mathrm{S}, 39^{\circ} 17^{\prime} \mathrm{W}$, maio 2007 (st.), G.S. Campos \& A.P. Alencar 54 (ALCB); Camacan, jan. 1966 (fl.), R.S. Pinheiro 5 (CEPEC); Canavieiras, out. 1988 (fr.), L.A.M. Silva et al. 2621 (CEPEC, HUEFS); Catu, dez. 1986 (fl.), A.S. Cerqueira et al. 4 (ALCB, CEPEC, HRB, HUEFS); Conde, 12 $02^{\prime} 32^{\prime \prime} \mathrm{S}$, 37\%43'21"W, out. 1995 (fl.), L.N. Silva \& M.C. Ferreira 90 (HRB); Cotegipe, out. 1986 (f1.), M.L. Guedes \& L.B. Silva 1169 (ALCB); Entre Rios, fev. 1999 (fr.), N.G. Jesus et al. s.n. (ALCB 43191); Igrapiúna, $13^{\circ} 48^{\prime} 08^{\prime \prime} \mathrm{S}, 39^{\circ} 10^{\prime} 03^{\prime \prime} \mathrm{W}$, dez. 2010 (fl.), J.L. Paixão \& L. Rocha 1838 (HUESC); Ilhéus, 14 ${ }^{\circ} 57^{\prime} 15^{\prime \prime} \mathrm{S}, 39^{\circ} 03^{\prime} 02^{\prime \prime W}$, ago. 2011 (fr.), R.O. Perdiz et al. 874. (CEPEC, HUEFS); Ipiaú, out. 1970 (fl.), T.S. Santos 1236 (CEPEC, HUEFS); Itabuna, abr. 1965 (fr.), R.P. Belém \& M. Magalhães 698 (CEPEC); Itacaré, 14¹8'S, 3859'W, mar. 1974 (fl.), R.M. Harley et al. 17564 (CEPEC); Itagibá, $14^{\circ} 00^{\prime} \mathrm{S}, 39^{\circ} 00^{\prime} \mathrm{W}$, jan. 2009 (fr.), M.L. Guedes et al. 16522 (ALCB); Itajuípe, $14^{\circ} 40^{\prime} \mathrm{S}, 39^{\circ} 30^{\prime} \mathrm{W}$, jan. 2003 (fl.), P. Fiaschi et al. 1260 (CEPEC); Itamaraju, nov. 1972 (fl.), M.T. Monteiro 23582 (HUEFS); Itanagra, maio 1981 (fl.), S.A. Mori \& B.M. Boom 14135 (CEPEC); Itapebi, nov. 1967 (fl.), R.S. Pinheiro \& T.S. Santos 386 (CEPEC); Ituberá, 1340'S, 39 $01^{\prime} \mathrm{W}$, dez. 2009 (fl.), C.A.C. Queiroz et al. 16 (HUEFS); Jussari, 1509'12"S, 39³1'50"W, mar. 2003 (fr.), W.W. Thomas et al. 13395 (CEPEC); Maraú, ago. 1967 (st.), S.G. Vinha \& R.S. Pinheiro 23 (CEPEC);

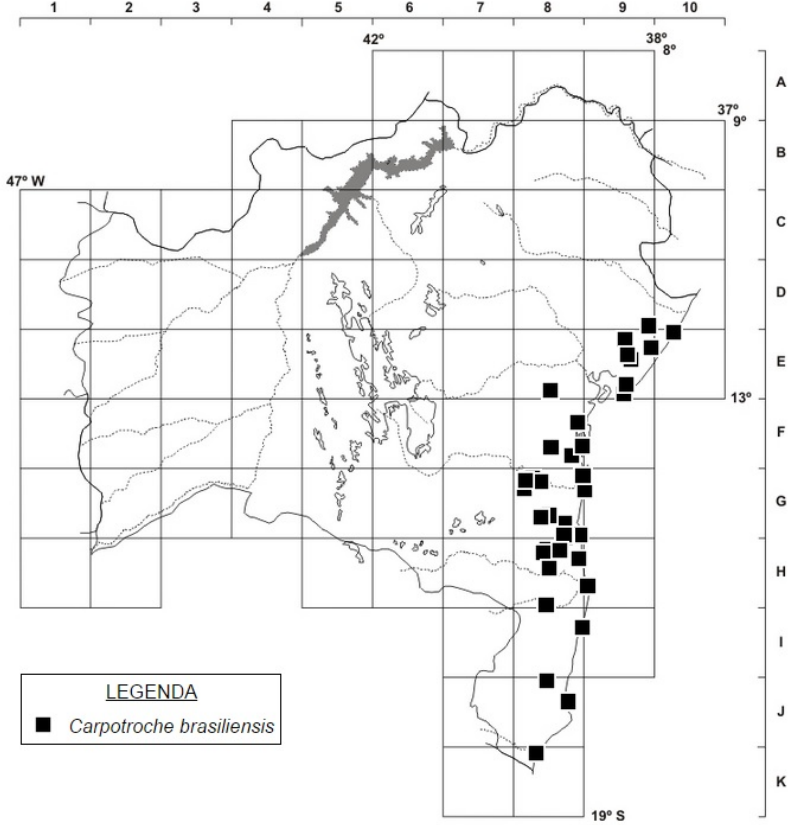

Figura 1. Mapa de distribuição de Carpotroche brasiliensis no estado da Bahia.

Mucuri, $18^{\circ} 05^{\prime} 01^{\prime \prime S}, 39^{\circ} 40^{\prime} 24^{\prime \prime W}$, out. 2000 (fr.), L.A.M. Silva et al. 4133 (HUESC); Pojuca, nov. 2006 (fr.), C.S. Silva-Lima et al. 99 (ALCB, HRB, HUEFS); Potiraguá, 15²12'12"S, 39³4'30"W, ago. 2006 (fr.), J.L. Paixão et al. 1063 (CEPEC, HUESC); Prado, mar. 1978 (fr.), S.A. Mori et al. 9733 (CEPEC); Salvador, 1255'37"S, $38^{\circ} 25^{\prime} 37^{\prime \prime} \mathrm{W}$, out. 2003 (fl.), E.P. Queiroz 275 (HRB, HUEFS); Santa Cruz Cabrália, maio 1994 (fr.), M.L. Guedes et al. 3126 (ALCB); Santa Terezinha, 12 $52^{\prime} 11^{\prime \prime} \mathrm{S}, 3^{\circ} 28^{\prime} 37^{\prime \prime} \mathrm{W}$, out. 2003 (fl.), M.L.C. Neves 1 (HUEFS); Simões Filho, jul. 1988 (fr.), N.N.A. Santos s.n. (HUEFS 8607); Una, ago. 1999 (fr.), L.A.M. Silva et al. 4011 (ALCB, CEPEC, HUEFS, HUESC); Uruçuca, 14 ${ }^{\circ} 59^{\prime} \mathrm{S}$, $39^{\circ} 16^{\prime} \mathrm{W}$, maio 1994 (fr.), W.W. Thomas et al. 10406 (CEPEC);

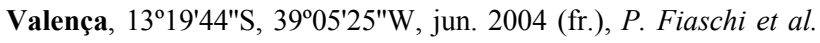
2375 (CEPEC); Wenceslau Guimarães, $13^{\circ} 41^{\prime} \mathrm{S}, 39^{\circ} 28^{\prime} \mathrm{W}$, dez. 2001 (fr.), L.J. Alves et al. 401 (ALCB).

Material adicional - BRASIL. MINAS GERAIS: Belo Horizonte, nov. 1941 (fl.), M. Barreto 11240 (HUEFS).

Caracteriza-se por possuir flores estaminadas e monoclinas em indivíduos diferentes e frutos com alas membranáceas a papiráceas, adpressas ao epicarpo e cobrindo parcialmente umas às outras.

\section{Kuhlmanniodendron Fiaschi \& Groppo}

Árvores ou arvoretas, poligâmicas. Folhas alternas, espiraladas; lâmina com margem inteira; venação broquidódroma; estípulas ausentes ou precocemente decíduas. Inflorescências botrioides, axilares. Flores unissexuadas, perianto assimetricamente arranjado. Sépalas 3, conatas na base. Pétalas 6 ou 7. Estames 14-40; filetes glabros; anteras oblongas, lineares ou lanceoladas, glabras. Ovário tricarpelar, 2-4 óvulos por carpelo, liso; estiletes 3; estigma em forma de "U". Frutos bagas, indeiscentes; epicarpo liso, estriado ou tuberculado. Sementes 1 a numerosas. 


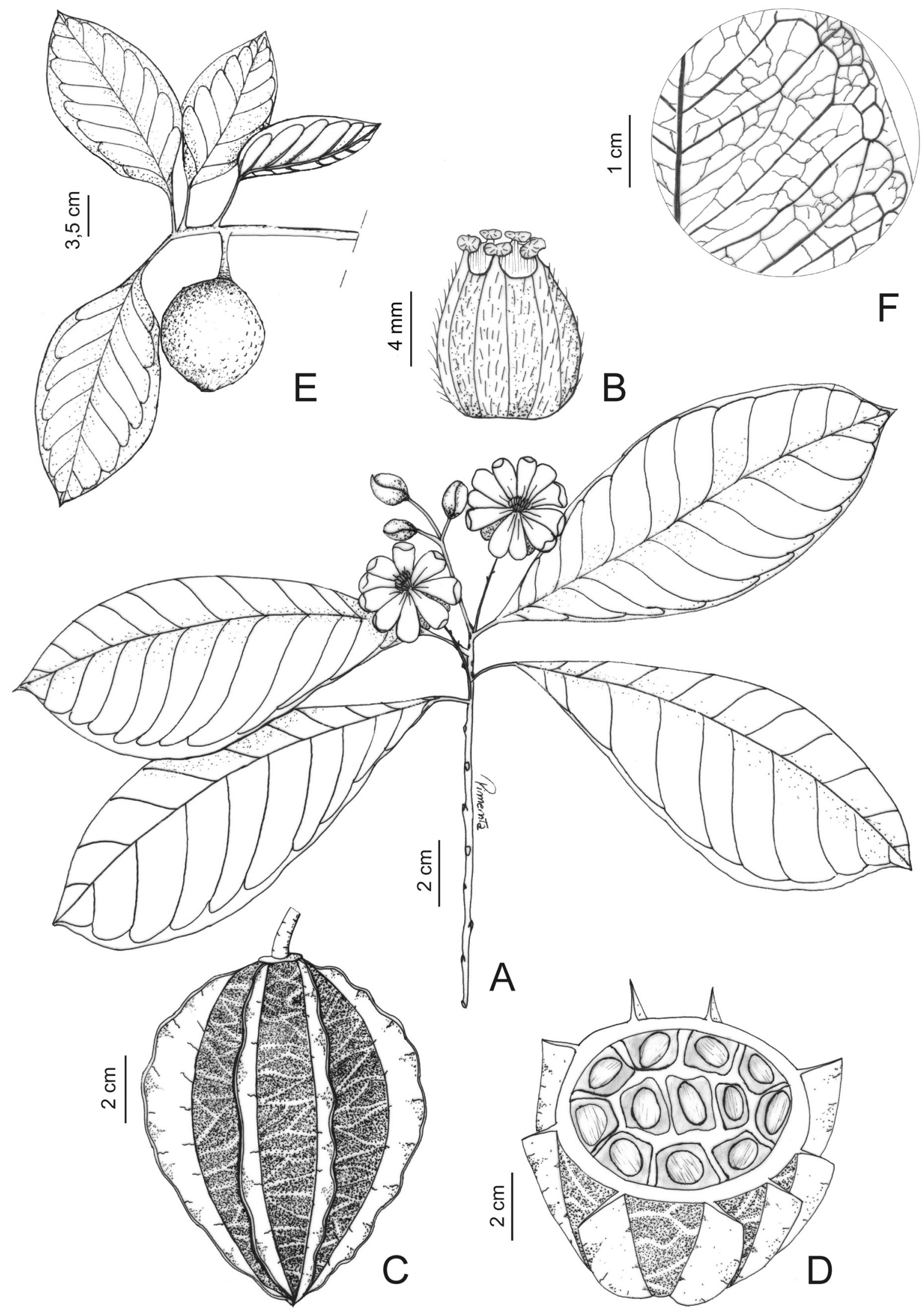

Figura 2. A-D. Carpotroche brasiliensis: A- ramo com flores estaminadas; B- pistilo; C- fruto fechado; D- fruto em corte transversal, evidenciando as sementes. E, F. Kuhlmanniodendron macrocarpum: E- ramo com fruto; F- folha com detalhe das nervuras na face abaxial (A- Cardoso 1564 HUEFS; B- Barreto 11240 HUEFS; C- Santos s/n HUEFS 8607; D- Paixão 1366 HUEFS; E- Guedes 16361 ALCB; F- Jardim 1117 CEPEC). 
Kuhlmanniodendron é endêmico do Brasil e composto por duas espécies, ocorrendo na Mata Atlântica da Bahia e do Espírito Santo (Fiaschi \& Groppo 2008; Groppo et al. 2013).

\subsection{Kuhlmanniodendron macrocarpum Groppo,} Favaretto \& Fiaschi, Syst. Bot. 38(1):167. 2013.

\section{Figuras 2E, F e 3.}

Árvores até $25 \mathrm{~m}$ alt., glabras; ramos levemente estriados longitudinalmente, geralmente com lenticelas esparsas. Folhas com pecíolo 3-7 cm compr.; lâmina 8-20,5 × 4,5-10 cm, elíptica a obovada, base atenuada a cuneada, ápice arredondado a agudo ou acuminado, cartácea a coriácea, verde intenso e brilhante adaxialmente, verde pálido e opaco abaxialmente, glabra, margem inteira, nervuras principal e secundárias planas adaxialmente, proeminentes abaxialmente, estípulas ausentes. Inflorescências com raque 3-8 cm compr.; pedicelo 5-12 $\mathrm{mm}$ compr. Flores estaminadas $12-18 \mathrm{~mm}$ diâm. Sépalas verrucosas. Pétalas 7-12 × 3-5 mm, livres, venação conspícua abaxialmente. Estames 30-40; filetes ca. 1 $\mathrm{mm}$ compr., conatos na base, organizados externamente em um anel e internamente em conjuntos de dois ou três; anteras 5-7 $\mathrm{mm}$ compr., lineares a lanceoladas. [Flores pistiladas não vistas]. Bagas 5-8 $\times$ 4-6 cm, elípticas a esféricas, com estiletes persistentes, epicarpo liso a levemente tuberculado. Sementes numerosas, $8-15 \times 5-12 \mathrm{~mm}$, 4-anguladas ou clavadas, com tricomas nos ângulos.

Endêmica da Bahia (Groppo et al. 2013). F8, G8: Mata Atlântica, em florestas estacionais semideciduais, florestas ombrófilas densas e matas higrófilas no sul da Bahia. Encontrada com flores em janeiro e setembro e com frutos de março a julho.

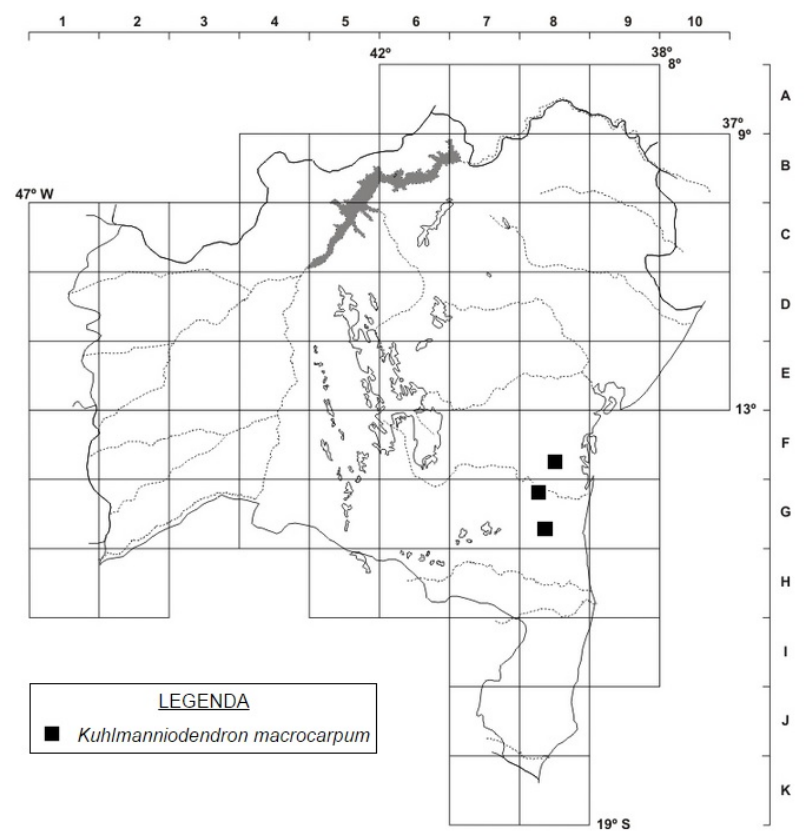

Figura 3. Mapa de distribuição de Kuhlmanniodendron macrocarpum no estado da Bahia.

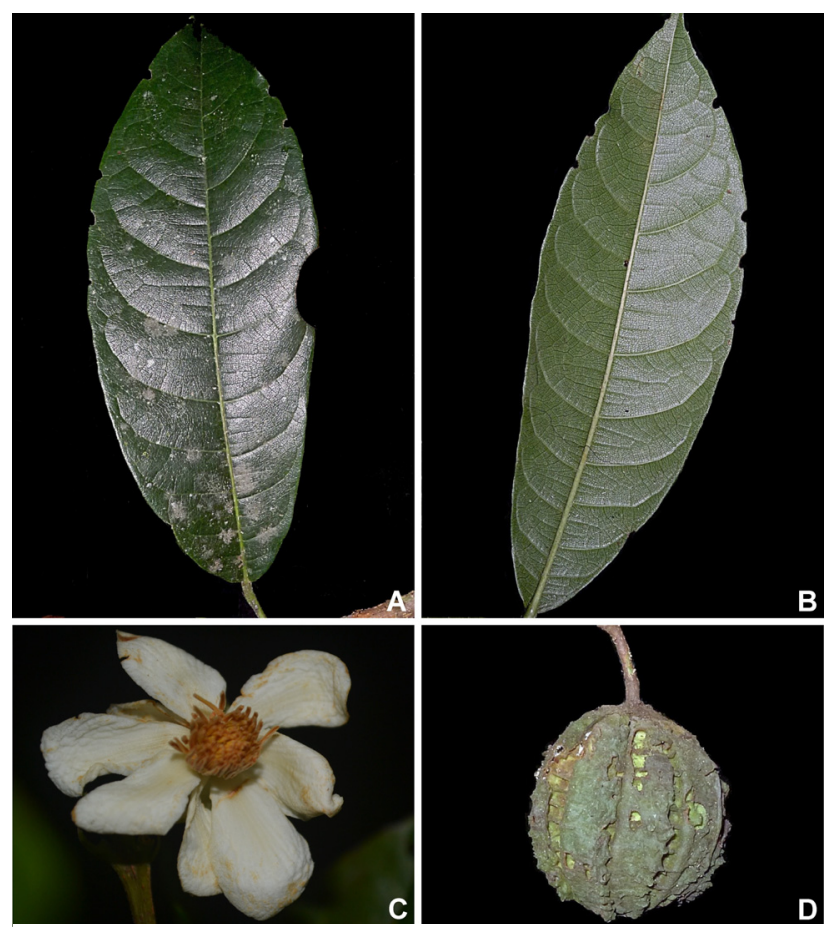

Figura 4. A-D. Carpotroche brasiliensis: A- folha (face adaxial); B- folha (face abaxial); C- flor estaminada; D- fruto (Fotos: A, B, D- Alex Popovkin; C- Moabe Fernandes).

Material selecionado - Almadina, $14^{\circ} 44^{\prime} 06^{\prime \prime} \mathrm{S}, 39^{\circ} 04^{\prime} 46^{\prime \prime} \mathrm{W}$, jan. 2008 (fl.), J.G. Jardim et al. 5187 (CEPEC); Gandu, out. 1970 (fl.), T.S. Santos 1182 (CEPEC); Itagibá, 140'10'35"S, 3943'55"W, maio 2008 (fr.), C.E. Ramos \& L.J. Alves 194 (holótipo ALCB).

Caracteriza-se pelos filetes glabros e conatos na base e pelos frutos indeiscentes com epicarpo liso a levemente tuberculado. A descrição das flores foi retirada de Groppo et al. (2013).

\section{AgRADECIMENTOS}

Agradecemos ao Programa de Pesquisa em Biodiversidade (PPBIO) do Semiárido, ao Sistema Nacional de Pesquisa em Biodiversidade (SISBIOTA CNPq 563084 / 2010-3 e FAPESB PES0053 / 2011) e ao Reflora (CNPq 563546 / 2010-7 e 563548 / 2010-0 e FAPESB PES0054 / 2011) pelo financiamento das visitas aos herbários.

\section{REFERÊNCIAS}

Fiaschi, P. \& Groppo, M. 2008. Kuhlmanniodendron Fiaschi \& Groppo, a new eastern Brazilian genus of Achariaceae sensu lato segregated from Carpotroche Endl. (formerly included in Flacourtiaceae). Botanical Journal of the Linnean Society 157: 103-109.

Groppo, M.; Favaretto, B.S.G.; Silva, C.I.; Jardim, J.G. \& Fiaschi, P. 2013. A new species of Kuhlmanniodendron (Lindackerieae, Achariaceae) from Eastern Brazil and the systematic position of the genus in Achariaceae. Systematic Botany 38: 162-171. 
Marquete, R.; Torres, R.B. \& Medeiros, E.S. 2015. Achariaceae. In: Lista de Espécies da Flora do Brasil. Jardim Botânico do Rio de Janeiro. Disponível em http://floradobrasil.jbrj.gov.br/ jabot/floradobrasil/FB34; acesso em 15 maio 2015.
Sleumer, H.O. 1980. Flacourtiaceae. In: Flora Neotropica. Vol. 22. The New York Botanical Garden, New York.

\section{LISTA DE EXSICATAS}

Alves, L.J. 401 (1.1); Belém, R.P. 563, 632, 698, 1117, 1129 (1.1); Barreto, M. 11240 (1.1); Brito, H.S. 170 (1.1); Campos, G.S. 54 (1.1); Cardoso, D. 1564 (1.1); Carvalho, A. M. 1140 (1.1); Cerqueira, A.S. 4 (1.1); Costa, L.C.B. 180 (1.1); Eupunino, A. 227 (1.1); Ferreira, M.C. 753 (1.1); Fiaschi, P. 1260, 2375 (1.1); Guedes, M.L. 1169 (1.1), 1636 (2.1), 3201 (1.1),13126, 16361, 16522, PL0320 (1.1), s.n. ALCB 88086 (2.1); Hage, J.L. 1246 (1.1); Harley, R.M. 17564 (1.1); Jardim, J.G. 99 (1.1), 1117 (2.1) 2851 (1.1), 5187 (2.1); Jesus, N.G. PL766 (1.1); Jost, T. 171 (1.1); Leitman, P.M. 97 (1.1); Lima, S.S. 157 (1.1); Loureiro, D.M. 604 (1.1); Maas, P.J.M. 7071 (1.1); Magalhães, C.M. 72 (1.1); Marinho, L.C. 591 (1.1); Martini, A. 46 (1.1); Monteiro, M.T. 23582 (1.1); Mori, S.A. 9733, 11043, 14135 (1.1); Neves, M.L.C. 1 (1.1); Paixão, J.L. 1063, 1366, 1838 (1.1); Passos Jr., L.A. 838 (1.1); Perdiz, R.O. 874 (1.1); Pinheiro, R.S. 5, 386, 1190 (1.1); Queiroz, C.A.C. 16 (1.1); Queiroz, E.P. 275, 388 (1.1); Ramos, C.E. 104, 194 (2.1), 227 (1.1); Rylands, A. 9 (1.1); Sambuichi, R.H.R. 412, 560, 1164, s.n. HUEFS 41390 (1.1); Santana, D.L. 593 (1.1); Santos, N.N.A. (1.1); Santos, T.S. 330 (1.1), 1182 (2.1), 1236 (1.1); Silva, L.A.M. 1153, 1500, 2621, 4011, 4133, 5088 (1.1); Silva, L.N. 90 (1.1); Silva-Lima, C.S. 99 (1.1); Thomas, W.W. 10406, 13395 (1.1); Valadão, R.M. 400, 695, 742 (1.1); Vinha, S.G. 23 (1.1). 